$R M x A C, \mathbf{5 3}, 147-150(2021)$

(C) 2021: Instituto de Astronomía, Universidad Nacional Autónoma de México

https://doi.org/10.22201/ia.14052059p.2021.53.29

\title{
STELLAR EVOLUTION AND THEIR ASTRONOMICAL OBSERVATIONS
}

\author{
Aviral Pandey ${ }^{1}$ \\ RESUMEN
}

Hay un altísimo número de estrellas (unos pocos cientos de miles de millones) de diferentes edades, tamaños y masas en nuestra galaxia, la Vía Láctea y miles de millones de otras galaxias en el Universo. Fue extremadamente difícil para los astrónomos clasificar las estrellas en diferentes grupos para comprender sus propiedades con precisión. En general, las estrellas permanecen en las fases de la secuencia principal en el diagrama HR durante la mayor fracción de su tiempo de vida porque mantienen el equilibrio hidrostático durante esta fase. Las estrellas de diversa masa pasan a través de diferentes fases evolutivas. Algunos de estas estrellas terminan sus vidas como explosiones catastróficas que no se han entendido bien hasta ahora y tienen un gran potencial para comprender el proceso de evolución general de las estrellas y, a su vez, la evolución del Universo. Observaciones terrestres y espaciales de múltiples longitudes de onda de estos objetos son cruciales para comprenderlos en términos de las leyes de la física. En un futuro próximo, el avance de la tecnología exige un uso extensivo de inteligencia artificial, redes neuronales y robótica para comprender mejor las observaciones astronómicas.

\section{ABSTRACT}

There is a hugh number of stars ( few hundred billions) of different ages, sizes and masses in our galaxy, the Milky Way, and billions of other galaxies in the Universe. It was extremely challenging for astronomers to classify them into different groups to understand their properties precisely. In general, stars remains in the main sequence phases in the HR diagram for the largest fraction of its life time because it maintains hydro-static equilibrium during this phase. Stars of diverse mass range pass through different evolutionary phases. Some of these end their lives as catastrophic explosions not understood well so far and have a great potential to understand the overall evolution process of stars and in turn evolution of the Universe. Ground and space-based multi-wavelength observations of these objects are crucial to understand them in terms of laws of physics. In near future, advancement of technology demands extensive use of artificial intelligence, neural networks, robotics to understand astronomical observations in a better way.

Key Words: stars: evolution — supernovae: general — telescopes

\section{INTRODUCTION}

In the past, it was extremely challenging for astronomers to classify stars into different groups to understand their properties precisely. In late nineteenth century, astronomers had developed tools that can probe about stars with a great deal. In 1905, the Danish astronomer Einar Hertzsprung, and the American astronomer Henry Norris Russell, looked for the various properties of stars such as luminosity, effective temperatures, absolute magnitude and spectral classes Hertzsprung and Russell (HR) plotted the temperature of stars vs their luminosity. It is known that in the theoretical HR diagram neither luminosity nor effective temperature is a directly observed quantity. So, they also plotted the color of stars or spectral type vs. their absolute magnitude, known as the observational HR diagram because as both are the observational quantity and

\footnotetext{
${ }^{1}$ Brightlands School, 17-C, Raipur Road, Dalanwala, Dehradun, Uttarakhand 248001.
}

is also known as color-magnitude diagram of stars. They noticed that luminosity of stars decreased from spectral type $\mathrm{O}$ to $\mathrm{M}$. The HR diagram is the most facinating graphical tools to understand the stellar evolution. Each individual stars have different initial mass, hence each star goes through specific evolutionary stages. Each of these evolutionary stages could be understood from the HR diagram. They are basically due to change in the temperature and luminosity of the stars as the stars evolves with time. HR diagram in Figure 1 shows the different groups (Main sequence, Giants, Supergiants, White dwarfs) of the stars depending on how they produces energy.

\section{EVOLUTION OF STARS}

The eEvolution of stars means the way they change or evolve with time. It is now understood to a good extend that stars produce energy by nuclear fusion reactions that occur in the star core which is responsible for star formation processes. Stars form when a dense cloud of gas (called molecular clouds) 


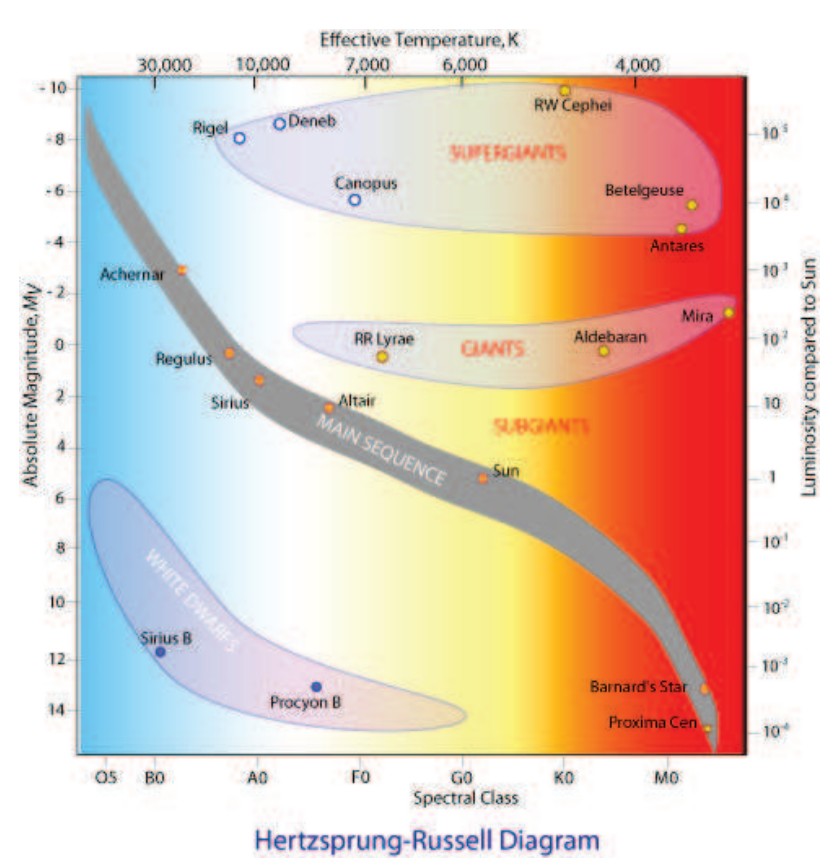

Fig. 1. Evolution of stars as understood in terms of HR diagram through properties like luminosity, temperature and spectral class. Credit: https://www.atnf.csiro.au/outreach/education/senior/ cosmicengine/stars_hrdiagram.html

collapses until nuclear reactions begin deep in the interior of the cloud and provide enough energy to halt the collapse, a situation called hydro-static equilibrium. A star remains in its main sequence of the HR diagram for the largest fraction of its life time because it maintains hydro-static equilibrium during this phase. It is also known that high mass stars have more fuel, therefore they emits more energy and evolve more rapidly than low mass stars, which emits less energy. Various phases of stars are broadly described as (also see Fig. 1 for more details):

(a) The main sequence phase: Just after taking birth through the collapse of inter-stellar dusts or giant molecular clouds, the stars reach in the main sequence, which is characterized by hydrogen fusion in their core. It means, during the main sequence phase, a star mainly burns (fuses) Hydrogen to form Helium.

(b) The Sub-Giant phase: Due to formation of He in the core. The core becomes He rich. At this stage the temperature is not high enough to sustain further fusion. But H-burning continues in a shell around the core and as the temperature increase the pressure outside the core increases and the $\mathrm{H}$-shell expands marking star entering in to the sub giant phase.

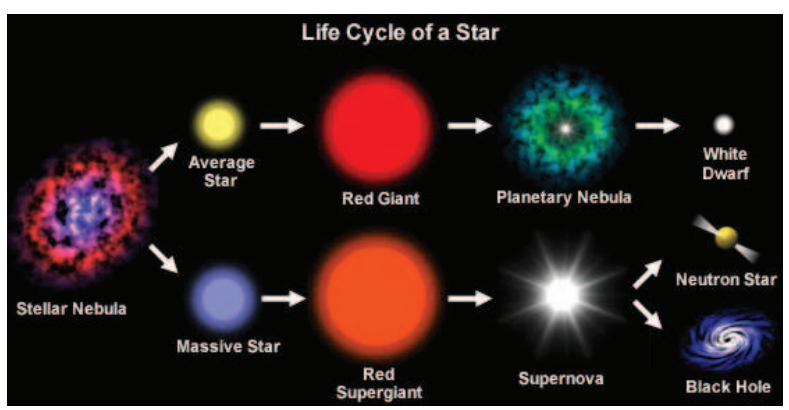

Fig. 2. A cartoon of the typical life cycle of low and high mass stars. Credit: https://scioly.org/wiki/index.php/Astronomy/Stellar Evolution.

(c) The Red-Giant phase: After the sub-giant phase, the core has enough temperature for fusing $\mathrm{He}$ to form carbon. The outer layers begin to expand, cool and shine less brightly. The expanding star is now called a Red-Giant.

(d) White Dwarf phase: The He core runs out and very soon the core consists only of Carbon and Oxygen. The CO core grows while a He-burning shell source develops. The outer layers begins to drift away from the core as a gaseous envelope.In fact there exists a super-wind which removes the envelope mass before the core has grown to its maximal possible size. This gas that surround the core is called a planetary Nebula. Now the remaining core, which is around $80 \%$ of the original star, approaches its final stage where the core becomes highly dense, compact, cool and dim called a white dwarf.

However, in case of high mass stars $(\sim>8 M \odot)$, The contracting core get sufficient temperature for carbon burning, and start to burn to neon. This process of core burning followed by core contraction and shell burning keep on going in a series of nuclear reactions producing successively heavier elements until iron is formed in the core. Iron cannot be burned to heavier elements because it is the most stable element. Finally, all the fuel of star run out and it begin collapses under its own gravity.

The mass of the core of the star allow what happens next. If the core has a mass less than about 3 solar mass, the collapse of stars may be stopped by the pressure of neutrons (this is an even more extreme state than the electron pressure that balance the white dwarfs). In this case, the core converted in a neutron star. If the core of star has a mass greater than about 3 solar masses, even neutron pressure is not sufficient to balance gravity, and it will collapse further into a black hole (see Fig. 2). 


\section{STELLAR EXPLOSIONS}

Stellar explosions in the Universe emits enormous amount of energy in form of energetic particles and radiation in almost all wavelengths: X-rays/gammarays/ infrared/ultraviolet/radio waves. They are transient astronomical events, occur during the final stage of stellar evolution. Massive stars during its evolutionary stages undergo a series of transitions from proto-star to a main sequence star and finally end up as a blue super-giant or a red super-giant star. At this stage, a series of nuclear fusion reactions take place and finally an inert iron core is left behind. Some of the important stellar explosions are:

\subsection{Supernovae}

Supernovae (SNe) are powerful stellar explosions and are responsible for enrichment of heavy elements in the present Universe. The word "Supernova" was given by Walter Baade and Fritz Zwicky in 1931. The classification of SNe is based on their different characteristics. SNe are broadly classified as Thermonuclear and Core-collapse Supernovae (CCSNe). A detailed classification is also provided based on the observational characteristics seen in their light curves and the presence of various chemical elements in their spectra (Weiler \& Sramek 1988; Smartt et al. 2009). A cartoon of a typical supernova explosion are also explained in Figure 3.

A supernova located close enough to our Earth can have much effect on its biosphere depending upon the energy and category of supernova. Near Earth-Supernovae can also cause global warming of around 3-4 celcius. Around 20 supernovae has been happened near to Earth (within 300pc) over last 11 million years.

\subsection{Gamma-ray bursts}

Gamma-ray bursts (GRBs) are intense flashes of gamma-rays which can last from a few milli-seconds to several hours. They observed from the sky in arbitrary directions and hence they are isotropic in nature. They are the brightest known class of stellar scale explosions known to occur in electromagnetic band in the Universe. GRBs have two distinct phases. Initial phase is known as the prompt emission phase which is short lived and usually gives emission in gamma-rays. GRBs are classified into two types based on two distinct peaks on their histogram, which are separated by a dip at 2 seconds of their duration measured in gamma-rays. Bursts having duration less than 2 sec are called as short GRBs and those that last for more than $2 \mathrm{sec}$ are known as long GRBs. Based only on the two peaks

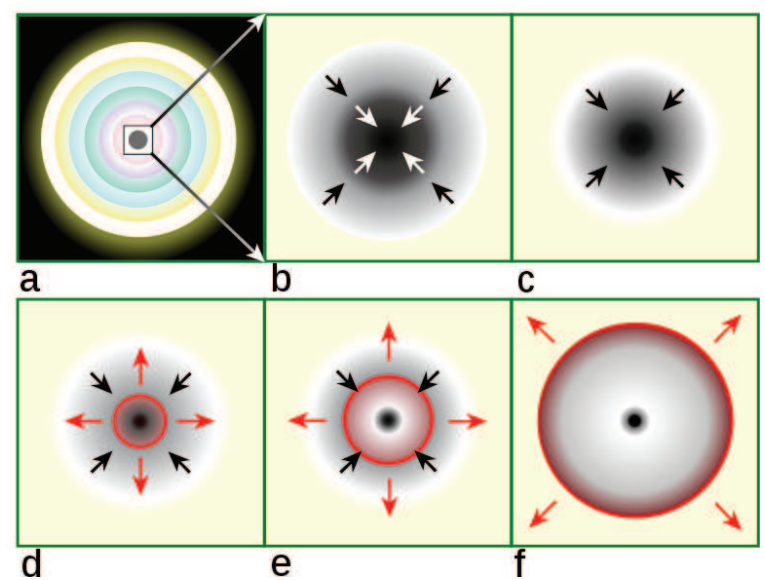

Fig. 3. A simple cartoon of the core collapse scenario of a massive star as reffered using web-tool https://en.wikipedia.org. In case of (a) A massive, evolved star has onion-layered shells of elements undergoing fusion. An inert iron core is formed from the fusion of Silicon in the inner-most shell. In (b), the iron core reaches Chandrasekhar-mass and starts to collapse, with the outer core (black arrows) moving at supersonic velocity (shocked) while the denser inner core (white arrows) travel sub-sonicallyi. In (c), the inner core compresses into neutrons and the gravitational energy is converted into neutrinos. In case of (d), the infalling material bounces off the nucleus and forms an outward-propagating shock wave (red). Whereas in (e), the shock begins to stall as nuclear processes drain energy away, but it is re-invigorated by interaction with neutrinos. Finally in (f), the material outside the inner core is ejected, leaving behind only a degenerate remnant. However, there could be other possible scenarios for such supernova explosions. Credit: https://en.wikipedia.org/wiki/Supernova\#/media/

in the duration distribution, and well before anything was known about the distance or environment of GRBs, it was imagined that these peaks related to two distinct progenitors. Recent observations confirm that long GRBs are possible outcome of the gravitational collapse of massive and rotating stars, since spectroscopic features from supernovae (SNe) have been detected in optical follow-up observations of GRBs. While short GRBs are possible outcome of mergers of compact objects in binary systems (It can be Neutron-Neutron stars or Neutron starBlack Hole system). Physical origin of these short explosion is still not fully understood. GRBs are generally followed by long-lasting afterglow phase which gives emission at longer wavelengths (X-ray, ultraviolet, optical, infrared, microwave and radio). Afterglow being longer-lasting than GRBs prompt emission, provide a multi-band platform to study 
these energetic cosmic explosions in detail. Afterglow light curves and their broadband spectral energy distributions (SEDs) are the two key features required to understand the properties of the GRB afterglow(Pandey 2013).

\section{TOOLS TO UNDERSTAND STELLAR EXPLOSIONS}

Stellar explosions are time critical in nature and demand very fast response to observe them at high cadence to understand their nature or to trigger bigger facilities for spectroscopic follow-up observations. Network of small robotic optical telescopes such as the Burst Observer and Optical Transient Exploring System (BOOTES) can play a very significant role to quick (some of the data being contemporaneous to the prompt emission) and long follow-up observations of such explosions (Castro-Tirado et al 2011) in near future. MASTER group of robotic telescopes (http://observ.pereplet.ru/) also play improtant role in discovering new kind of trasients (Lipunov et al 2010). Hands on experience with BOOTES network fo telescopes is a very good initiative to learn about transients in general.

Machine learning techniques to use big data sets from the upcoming survey telescopes is another good way to learn new methods and evolve strategies to study about various types of stellar explosions is another potential area to work with. Many groups such as Pan-STARRS1 have recently started using machine learning to improve our understanding about astronomical transients (Wright 2015).

In near future, using photometric data of various types of astronomical transients from survey facilities an approach like The Photometric LSST Astronomical Time-Series Classification Challenge (PLAsTiCC) could be another potential way to hit this big research field and understand transients in a more systematic way $^{2}$.

\section{SUMMARY}

Studies about stellar evolution provide enormous information about the underlying physics and to understand energetic trasients in a much broader way. Optical telescopes of various apertures and with the help of sofisticated detectors and software tools like machine learning, robotics, neural networks is a possible way to proceed ahead in near future to explore the field in a big way. To improve the accuracy overall, use of neural networks has started to catch-up fast recently(Tagliaferri 2003) among astronomical community. We hope that approach like PLAsTiCC in near future will be very helpful to understand stars and their evolution in a better way.

Acknowledgments: I sincerely acknowledge kind support and guidance of Dr. S. B. Pandey ARIES Nainital during my short visits to ARIES to learn about basics of astronomy and its applications to understand evolution of stars and their observations. During my holidays from collage schedule, I really enjoyed working with my own small telescope to explore the sky in dark nights of Nainital under kind guidance of Dr. Pandey and it has been a great learning experience for my future endevours. I also sincerely thank to provide me an opportunity to remotely present my thoughts as a poster during AstroRob-2019 in such a early stage of my career.

\section{REFERENCES}

Castro-Tirado, A. J. 2011, AcPol, 51, 16

Lipunov, V., Kornilov, V., Gorbovskoy, E., et al. 2010, AdAst, 30L

Pandey, S. B. 2013, JApA, 34, 157

Smartt, S. J. 2009, ARA\&A, 47, 63

Tagliaferri, R., Longo, G., Andreon, S., et al. 2003, LNCS, 2859, 226

Weiler, K. W. \& Sramek, R. A. 1988, ARA\&A, 26, 295

Wright, D. E., Smartt, S. J., Smith, K. W., et al. 2015, MNRAS, 449, 451

\footnotetext{
${ }^{2}$ https://plasticc.org/.
} 